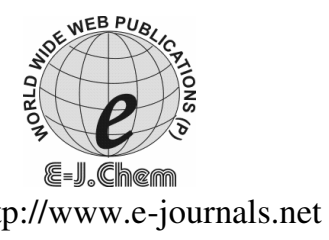

ISSN: 0973-4945; CODEN ECJHAO

E-Journal of Chemistry 2010, 7(2), 465-472

\title{
Molecular Interaction Studies on $N$-Alkanols with DMA in Toluene at 303K
}

\author{
S. THIRUMARAN* ${ }^{*}$ J. EARNEST JAYAKUMAR and \\ B. HUBERT DHANASUNDARAM \\ *Department of Physics (DDE), \\ Annamalai University, Annamalainagar- 608 002, India. \\ thirumaran64@gmail.com
}

Received 17 September 2009; Accepted 10 November 2009

\begin{abstract}
The ultrasonic study of velocity, density and viscosity has been measured for the mixtures of $n$-alkanols, namely; 1-propoanol, 1-butanol and 1-pentanol in toluene with $N-N$ dimethyl acetamide (DMA) at $303 \mathrm{~K}$. The experimental data have been used to calculate the acoustical parameters such as adiabatic compressibility $(\beta)$, intermolecular free length $\left(\mathrm{L}_{\mathrm{f}}\right)$, free volume $\left(\mathrm{V}_{\mathrm{f}}\right)$, internal pressure $\left(\pi_{\mathrm{i}}\right)$, and acoustic impedance $(\mathrm{Z})$. The excess values of the above parameters have also been evaluated and presented. From the present investigation, it is obvious that a weak molecular association was identified. Mixing of DMA with $n$-alkanols causes dissociation of hydrogen bonded structures of $n$-alkanols. Also, further addition of DMA with the mixture not only causes dissociation of hydrogen bonded structures of $n$-alkanols but also a decrease in molecular association between toluene and $n$-alkanols is observed. The evaluated excess values predict weak molecular interactions existing between DMA- $n$-alkanols as well as toluene- $n$-alkanols.
\end{abstract}

Keywords: Adiabatic compressibility, Free length, Hydrogen bonding, Dipole-dipole interactions, Viscous relaxation time

\section{Introduction}

In recent years, the measurement of ultrasonic velocity has been adequately employed in understanding the nature of molecular interactions in pure liquids and liquid mixtures. The ultrasonic velocity measurements are highly sensitive to molecular interactions and can be used to provide qualitative information about the physical nature and strength of molecular interaction in the liquid mixtures ${ }^{1-3}$. Ultrasonic velocity of a liquid is fundamentally related to the binding forces between the atoms or the molecules and has been adequately employed in understanding the nature of molecular interaction in pure liquids ${ }^{4-7}$, binary and ternary mixture. The variation of ultrasonic velocity and related parameters throw much light upon 
the structural changes associated with the liquid mixtures having weakly interacting components $^{8,9}$ as well as strongly interacting components. The study of molecular association in organic ternary mixtures having alcohol as one of the components is of particular interest, since alcohols are strongly self-associated liquid having a three dimensional network of hydrogen bond ${ }^{10}$ and can be associated with any other group having some degree of polar attractions ${ }^{11}$.

An exhaustive survey of literature has shown that a few attempts have been made for the ultrasonic velocity data of ternary mixtures ${ }^{12-14}$. However, no effort appears to have been made to collect the ultrasonic velocity data for the ternary mixtures of $\mathrm{N}, \mathrm{N}$-dimethylacetamide, in toluene with 1-alkanols. Hence, experimental study were carried out by the author and reported $\mathrm{N}, \mathrm{N}$-dimethylacetamide + touene +1 -propanol +1 -butanol, and 1-pentanol at $303 \mathrm{~K}$. The main purpose of this study is to characterize the molecular interactions in these systems and to subsequently evaluate the effect of chain length of 1 -alcohols on the ultrasonic velocity values of these mixtures.

The present ternary liquid systems taken for study at $303 \mathrm{~K}$ are

$\begin{array}{cccc}\text { System-I } & \text { 1-Propanol } & \text { + Toluene } & \text { + Dimethylacetamide (DMA) } \\ \text { System-II } & \text { 1-Butanol } & \text { + Toluene } & \text { + Dimethylacetamide (DMA) } \\ \text { System-III } & \text { 1-Pentanol } & \text { + Toluene } & \text { + Dimethylacetamide (DMA) }\end{array}$

\section{Experimental}

The chemicals used in the present work were analytical reagent (AR) and spectroscopic reagent (SR) grades with minimum assay were obtained from Sd fine chemicals India and E-merck, Germany as such without further purification. In all systems, the various concentrations of the ternary liquid mixtures were prepared in terms of mole fraction, out of which the mole fraction of the second component toluene $\left(\mathrm{X}_{2}=0.4\right)$ was kept fixed while the mole fractions of the remaining two components 1-alkanols $\left(\mathrm{X}_{1}\right)$ and DMA $\left(\mathrm{X}_{3}\right)$ were varied from 0.0 to 0.6 . There is nothing significant in fixing the second component at 0.4 . The density of pure liquids and liquid mixtures were determined using a specific gravity bottle by relative measurement method with an accuracy of $\pm 0.1 \mathrm{mg}$ (Model: SHIMADZU AX-200).An Ostwald's viscometer which is $10 \mathrm{~mL}$ capacity was used for the viscosity measurement of pure liquids and liquid mixtures. The viscometer was calibrated with fresh conductivity water immersed in the water bath which was kept at the experimental temperature. The time of flow $\left(t_{w}\right)$ of water and the time flow $\left(t_{s}\right)$ of solution was measured with digital stop clock having an accuracy of $0.01 \mathrm{~s}$ (Model: RACER HS-10 W) An ultrasonic interferometer (Model: F81) supplied by M/s. Mittal Enterprises, New Delhi, having the frequency $3 \mathrm{MHz}$ with an overall accuracy of $\pm 2 \mathrm{~ms}^{-1}$ has been used for velocity measurement.

\section{Theory}

Using the measured data, the following acoustical parameters have been calculated Adiabatic Compressibility

$$
\beta=\frac{1}{U^{2} \rho}
$$

Intermolecular free length $\left(\mathrm{L}_{\mathrm{f}}\right)$ has been calculated from the following relation,

$$
\mathrm{L}_{\mathrm{f}}=\mathrm{K}_{\mathrm{T}} \sqrt{ } \beta
$$

Where, $K_{T}$ is a temperature dependent constant. Free volume $\left(V_{f}\right)$ has been calculated from relation, 


$$
V_{f}=\left[\frac{M_{e f f} U}{K \eta}\right]^{3 / 2}
$$

Where, $M_{\text {eff }}$ is the effective molecular weight $\left(M_{\text {eff }}=\Sigma \mathrm{m}_{\mathrm{i}} \mathrm{x}_{\mathrm{i}}\right.$, in which $\mathrm{m}_{\mathrm{i}}$ and $\mathrm{x}_{\mathrm{i}}$ are the molecular weight and the mole fraction of the individual constituents respectively). $\mathrm{K}$ is a temperature independent constant which is equal to $4.28 \times 10^{9}$ for all liquids.

The internal pressure $\left(\pi_{\mathrm{i}}\right)$ can be found out as

$$
\pi_{i}=b R T\left(\frac{K \eta}{U}\right)^{1 / 2}\left(\frac{\rho^{2 / 3}}{M_{e f f}^{7 / 6}}\right)
$$

$\mathrm{K}$ is a constant, $\mathrm{T}$ the absolute temperature, $\eta$ the viscosity in $\mathrm{Nsm}^{-2}$, $\mathrm{U}$ the ultrasonic velocity in $\mathrm{ms}^{-1}, \rho$ the density in $\mathrm{Kgm}^{-3}, M_{\text {eff }}$ the effective molecular weight. Excess parameter $\left(\mathrm{A}^{\mathrm{E}}\right)$ has been calculated by using the relation

$$
A^{E}=A_{\exp }-A_{i d}
$$

where $A_{i d}=\sum_{i=1}^{n} A_{i} X_{i}, A_{i}$ is any acoustical parameters and $\mathrm{X}_{\mathrm{i}}$ the mole fraction of the liquid component.

\section{Results and Discussion}

The experimentally determined values of the density $(\rho)$, viscosity $(\eta)$ and ultrasonic velocity $(\mathrm{U})$ at $303 \mathrm{~K}$ of all the pure components are listed in Table 1 , whereas Table 2 summarize the same for all the three ternary systems. The values of adiabatic compressibility $(\beta)$, intermolecular free length $\left(\mathrm{L}_{\mathrm{f}}\right)$, free volume $\left(\mathrm{V}_{\mathrm{f}}\right)$, internal pressure $\left(\pi_{\mathrm{i}}\right)$, and acoustic impedance $(Z)$ of the three liquid systems are furnished in Table 3 . The excess values of the above parameters have been evaluated and presented in the Table 4.

Table 1. Density $(\rho)$, viscosity $(\eta)$ and Ultrasonic velocity $(U)$ of pure liquids.

\begin{tabular}{cccc}
\hline Liquids & $\begin{array}{c}\text { Density } \\
\rho / \mathrm{kg} / \mathrm{m}^{3}\end{array}$ & $\begin{array}{c}\text { Viscosity } \\
\eta / \times 10^{-3} \mathrm{NSm}^{-2}\end{array}$ & $\begin{array}{c}\text { Velocity } \\
\mathrm{U} / \mathrm{m} / \mathrm{s}\end{array}$ \\
\hline 1-Propanol & 793.25 & 1.6075 & 1184.9 \\
1-Butanol & 799.62 & 2.1025 & 1218.7 \\
1-Pentonol & 805.60 & 2.6875 & 1251.6 \\
Toluene & 851.65 & 0.5729 & 1276.2 \\
$N-N$ Dimethylacetamide & 930.25 & 0.8991 & 1432.0 \\
\hline
\end{tabular}

In all the three ternary liquid systems, the ultrasonic velocity decreases with increasing concentration of 1-alkanols. This behaviour at such concentrations is different from the ideal mixtures behaviour can be attributed to intermolecular interactions in the systems studied ${ }^{15-16}$. The variation of ultrasonic velocity in a mixture depends upon the increase (or) decrease of intermolecular free length after mixing the components.

On the basis of a model, for sound propagation proposed by Eyring and Kincaid ${ }^{17}$, ultrasonic velocity should decrease, if the intermolecular free length increase and vice-versa. This is observed in the present investigation for all the three liquid systems. Such a decrease in ultrasonic velocity $(\mathrm{U})$ in all the three liquid systems with increasing molar concentration of alcohols which is attributed to, as the number of hydrocarbon group or chain-length of alcohol increases, a gradual decrease in sound velocity is noticed. This behaviour at such 
concentrations is different from the ideal mixtures behaviour can be attributed to intermolecular interactions in the systems studied ${ }^{15,16}$. The value of adiabatic compressibility $(\beta)$ however, shows an inverse behaviour as compared to the ultrasonic velocity $(\mathrm{U})$ i.e. the adiabatic compressibility $(\beta)$ increases with increase of concentration of alcohols in all the three liquid systems, which are tabulated in Table 3 . It is primarily the compressibility that increases due to structural changes of molecules in the mixture leading to a decrease in ultrasonic velocity ${ }^{18,19}$. Such a continuous increase in adiabatic compressibility with respect to the concentration of alkanols has been qualitatively ascribed to the effect of hydrogen bonding or dipole-dipole interactions ${ }^{20}$.

Table 2. Density $(\rho)$, viscosity $(\eta)$ and Ultrasonic velocity $(U)$.

\begin{tabular}{|c|c|c|c|c|}
\hline \multicolumn{2}{|c|}{ Mole Fraction } & \multirow{2}{*}{$\begin{array}{l}\text { Density } \\
\rho / \mathrm{kg} / \mathrm{m}^{3}\end{array}$} & \multirow{2}{*}{$\begin{array}{c}\text { Viscosity } \\
\eta / \times 10^{-3} \mathrm{NSm}^{-2}\end{array}$} & \multirow{2}{*}{$\begin{array}{c}\text { Velocity } \\
\mathrm{U} / \mathrm{m} / \mathrm{s}\end{array}$} \\
\hline $\mathrm{X}_{1}$ & $\mathrm{X}_{3}$ & & & \\
\hline \multicolumn{5}{|c|}{ System-I - 1-Propanaol + toluene + dimethylacetamide (DMA) } \\
\hline 0.0000 & 0.6001 & 819.6 & 0.6706 & 1269.6 \\
\hline 0.0998 & 0.5002 & 814.3 & 0.6958 & 1263.7 \\
\hline 0.2000 & 0.4000 & 809.8 & 0.7154 & 1257.6 \\
\hline 0.3000 & 0.3000 & 807.4 & 0.7390 & 1250.8 \\
\hline 0.3999 & 0.2000 & 804.2 & 0.7524 & 1244.7 \\
\hline 0.5000 & 0.1000 & 800.91 & 0.7732 & 1239.6 \\
\hline 0.6001 & 0.0000 & 787.87 & 0.7995 & 1230.3 \\
\hline \multicolumn{5}{|c|}{ System-II- 1-Butanol + toluene + dimethylacetamide (DMA) } \\
\hline 0.0000 & 0.5999 & 827.6 & 0.7944 & 1279.8 \\
\hline 0.0998 & 0.5000 & 822.9 & 0.8842 & 1272.4 \\
\hline 0.1998 & 0.4004 & 820.7 & 0.9242 & 1265.7 \\
\hline 0.3035 & 0.2918 & 816.8 & 0.9676 & 1259.4 \\
\hline 0.4000 & 0.2000 & 810.7 & 0.9776 & 1256.4 \\
\hline 0.5000 & 0.0998 & 806.3 & 1.0101 & 1249.6 \\
\hline 0.6001 & 0.0000 & 800.22 & 1.1010 & 1241.4 \\
\hline \multicolumn{5}{|c|}{ System-III - 1-Pentanol + toluene + dimethylacetamide (DMA) } \\
\hline 0.0000 & 0.5999 & 827.6 & 0.7944 & 1279.8 \\
\hline 0.0998 & 0.5000 & 822.9 & 0.8842 & 1272.4 \\
\hline 0.1998 & 0.4004 & 820.7 & 0.9242 & 1265.7 \\
\hline 0.3035 & 0.2918 & 816.8 & 0.9676 & 1259.4 \\
\hline 0.4000 & 0.2000 & 810.7 & 0.9776 & 1256.4 \\
\hline 0.5000 & 0.0998 & 806.3 & 1.0101 & 1249.6 \\
\hline 0.6001 & 0.0000 & 800.22 & 1.1010 & 1241.4 \\
\hline
\end{tabular}

Alkanols are liquids which are associated through the hydrogen bonding and in the pure state, they exhibit an equilibrium between the monomer and multimer species. Also, they can be associated with any other groups having some degree of polar attractions ${ }^{21}$. Similarly, $N-N$ dimethylacetamide (DMA) is belonging to the amide group which is highly polar, aprotic solvent and its molecules are associated through dipolar interactions ${ }^{22}$ Mixing of DMA with alkanols would release the dipoles of alkanols due to the breaking of hydrogen bonds.

As a result, the free dipoles released from the alkanols in association with DMA molecules forming strong hydrogen bonds, hence stronger molecular association existing between the DMA and alkanols molecules through hydrogen bonding ${ }^{22}$. Further, mixing of such DMA with 1-alkanols causes dissociation of hydrogen bonded structure of 1-alkanols and 
subsequent formation of (new) $\mathrm{H}$-bond $(\mathrm{C}=\mathrm{O}-\mathrm{H} \ldots . . \mathrm{O})$ between proton acceptor oxygen atom (with lone pair of electrons) of $\mathrm{C}=\mathrm{O}$ group of DMA and proton of $\mathrm{OH}$ - group of 1-alkanols ${ }^{23}$.

Table 3. Adiabatic compressibility $(\beta)$, free length $\left(\mathrm{L}_{\mathrm{f}}\right)$ free volume $\left(\mathrm{V}_{\mathrm{f}}\right)$, internal pressure $\left(\pi_{\mathrm{i}}\right)$ and Acoustic impedance $(\mathrm{Z})$.

\begin{tabular}{|c|c|c|c|c|c|c|}
\hline \multicolumn{2}{|c|}{ Mole Fraction } & \multirow{2}{*}{ 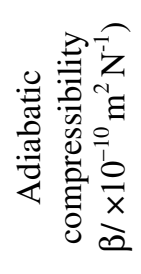 } & \multirow{2}{*}{ 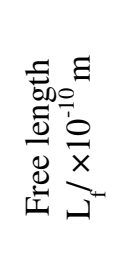 } & \multirow{2}{*}{ 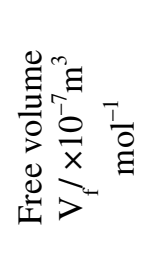 } & \multirow{2}{*}{ 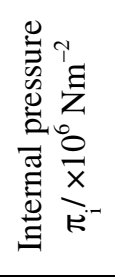 } & \multirow{2}{*}{ 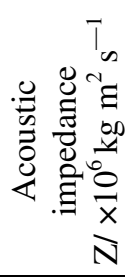 } \\
\hline $\mathrm{X}_{1}$ & $X_{3}$ & & & & & \\
\hline \multicolumn{7}{|c|}{ System-I - 1-Propanaol + toluene + dimethylacetamide (DMA) } \\
\hline 0.0000 & 0.6001 & 7.5694 & 0.5484 & 2.4750 & 111.37 & 1.0627 \\
\hline 0.0998 & 0.5002 & 7.6900 & 0.5533 & 2.2208 & 117.35 & 1.0290 \\
\hline 0.2000 & 0.4000 & 7.8079 & 0.5575 & 2.0165 & 118.17 & 1.0184 \\
\hline 0.3000 & 0.3000 & 7.9165 & 0.5614 & 1.8137 & 120.47 & 1.0099 \\
\hline 0.3999 & 0.2000 & 8.0261 & 0.5653 & 1.6555 & 121.91 & 1.0009 \\
\hline 0.5000 & 0.1000 & 8.1257 & 0.5688 & 1.5072 & 123.89 & 0.9928 \\
\hline 0.6001 & 0.0000 & 8.3850 & 0.5778 & 1.3418 & 126.68 & 0.9692 \\
\hline \multicolumn{7}{|c|}{ System-II - 1- Butanol + toluene + dimethylacetamide (DMA) } \\
\hline 0.0000 & 0.5999 & 7.3773 & 0.5419 & 1.9429 & 121.52 & 1.0592 \\
\hline 0.0998 & 0.5000 & 7.5059 & 0.5466 & 1.6043 & 130.31 & 1.0471 \\
\hline 0.1998 & 0.4004 & 7.6059 & 0.5503 & 1.4583 & 135.55 & 1.0388 \\
\hline 0.3035 & 0.2918 & 7.7189 & 0.5544 & 1.3186 & 141.26 & 1.0287 \\
\hline 0.4000 & 0.2000 & 7.8141 & 0.5332 & 1.2652 & 144.08 & 1.0186 \\
\hline 0.5000 & 0.0998 & 7.9426 & 0.5623 & 1.1668 & 148.89 & 1.0076 \\
\hline 0.6001 & 0.0000 & 8.1089 & 0.5682 & 0.9871 & 158.05 & 0.9933 \\
\hline \multicolumn{7}{|c|}{ System-III - 1-Propanaol + toluene + dimethylacetamide (DMA) } \\
\hline 0.0000 & 0.6099 & 7.2872 & 0.5386 & 1.7962 & 123.69 & 1.0672 \\
\hline 0.0999 & 0.5000 & 7.3899 & 0.5421 & 1.6797 & 127.37 & 1.0577 \\
\hline 0.2010 & 0.3971 & 7.4990 & 0.5464 & 1.6096 & 128.52 & 1.0465 \\
\hline 0.3000 & 0.3000 & 7.5922 & 0.5472 & 1.5528 & 129.57 & 1.0376 \\
\hline 0.4000 & 0.1997 & 7.6994 & 0.5536 & 1.4724 & 131.50 & 1.0286 \\
\hline 0.4900 & 0.0998 & 7.8146 & 0.5578 & 1.0880 & 149.49 & 1.0183 \\
\hline 0.6000 & 0.0000 & 7.9372 & 0.5621 & 0.9806 & 149.55 & 1.0092 \\
\hline
\end{tabular}

As 1-alkanols are associated through hydrogen bonding.

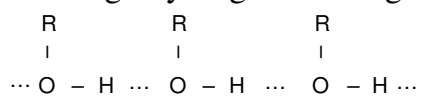

Further, the addition of $N, N$-Dimethylacetamide (DMA)) with mixtures causes dissociation of hydrogen bonded structure of 1-alkanols as well as decrease in interaction between toluene and alkanols. The subsequent formation of new hydrogen bonding between proton acceptor oxygen atoms of ${ }_{,}^{\prime}=0$ group of DMA and proton of - $\mathrm{OH}$ group of 1-alkanols ${ }^{20}$. $[\mathrm{C}=\mathrm{O} \cdots \mathrm{H}-\mathrm{O}-]$. 


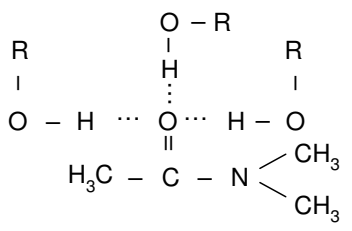

The same trend was observed in free length too in all the three systems, which are tabulated in Table 3. The increase in free length in all the three liquid systems is due to the loose packing of the molecules inside the shield, which may be brought by weakening of molecular interactions ${ }^{24}$.

Further, a decrease in free volume $\left(\mathrm{V}_{\mathrm{f}}\right)$ and an increase in internal pressure $\left(\pi_{\mathrm{i}}\right)$ with increase in concentration of the primary alcohols in all the three liquid systems is observed, which are listed in Table 3. Such an increase in internal pressure generally indicates association through hydrogen bonding ${ }^{25}$ and hence supports the present investigation. Similar results were observed by earlier workers ${ }^{14}$ in which an increase in internal pressure generally indicates association through hydrogen bonding.

Further, in all three liquid systems, the values of acoustic impedance $(Z)$ were found to be decreased, which are listed in Table 3. When an acoustic wave travels in a medium, there was a variation of pressure from particle to particle. The ratio of the instantaneous pressure excess at any particle of the medium to the instantaneous velocity of that particle is known as 'specific acoustic impedance' of the medium. This factor is governed by the inertial and elastic properties of the medium. It is important to examine specific acoustic impedance in relation to concentration and temperature. When a plane ultrasonic wave is set up in a liquid, the pressure and hence density and refractive index show specific variations with distance from the source along the direction of propagation. In the present investigation, it was observed that these acoustic impedance $(Z)$ values decrease with increasing concentration of $n$-alkanols. Such a decreasing values of acoustic impedance $(Z)$ further supports the possibility of molecular interactions between the unlike molecules.

In order to understand the nature of molecular interactions between the components of the liquid mixtures, it is of interest to discuss the same in terms of excess parameter rather than actual values. Non-ideal liquid mixtures show considerable deviation from linearity in their physical behaviour with respect to concentration and these have been interpreted as arising from the presence of strong or weak interactions. The effect of deviation depends upon the nature of the constituents and composition of the mixtures.

The excess values of adiabatic compressibility $\left(\beta^{\mathrm{E}}\right)$, intermolecular free length $\left(\mathrm{L}_{\mathrm{f}}^{\mathrm{E}}\right)$, free volume $\left(\mathrm{V}_{\mathrm{f}}^{\mathrm{E}}\right)$ and internal pressure $\left(\pi_{\mathrm{i}}^{\mathrm{E}}\right)$ for all the three liquid systems are furnished in Table 4. In the present investigation, it is observed that excess values of adiabatic compressibility $\left(\beta^{\mathrm{E}}\right)$ and excess free length $\left(\mathrm{L}_{\mathrm{f}}^{\mathrm{E}}\right)$ exhibit positive deviation over the entire range of composition in all three liquid systems.

It is learnt that negative values of $\beta^{\mathrm{E}}$ is an indication of strong intermolecular interactions in the liquid mixtures, which is attributed to charge transfer, dipole-induced dipole and dipole-dipole interactions and hydrogen bonding. While positive excess values to weak interactions which result from dispersion forces Rastogi ${ }^{26}$ from his studies of excess thermodynamic function in ternary mixtures has shown that the addition of third component weakens the strength of the interaction and the ternary mixture tends to approach ideal behaviour. Further $^{27}$, it is also suggested that the negative excess compressibility has been due to a closed packed molecules and positive excess values to weak interaction between unlike 
molecules. The Perusal of Table 4 shows the variation of the excess free volume as a function of concentration in all systems. The increasing values of free volume in all the systems with decreasing concentration of DMA resulting in weakening of hydrogen bonding interaction between unlike molecules. The negative values of excess internal pressure $\left(\pi_{\mathrm{i}}^{\mathrm{E}}\right)$ in all the three liquid systems which are furnished in Table 4 clearly confirm this prediction. Further, it is again well supported by the excess values of acoustic impedance $\left(\mathrm{Z}^{\mathrm{E}}\right)$ which are tabulated in Table 4 are all negative in all the liquid systems over the entire range of composition. The negative excess values of $\mathrm{Z}^{\mathrm{E}}$ clearly suggest that there is a weak molecular interaction $^{32}$ existing between the unlike molecules.

Table 4. Excess values of adiabatic compressibility $\left(\beta^{\mathrm{E}}\right)$, free length $\left(\left(\mathrm{L}_{\mathrm{F}}^{\mathrm{E}}\right)\right)$ free volume $\left(\mathrm{V}_{\mathrm{f}}^{\mathrm{E}}\right)$, internal pressure $\left(\pi_{\mathrm{i}}^{\mathrm{E}}\right)$ and acoustic impedance $\left(\mathrm{Z}^{\mathrm{E}}\right)\left(\mathrm{Z}^{\mathrm{E}}\right)$.

\begin{tabular}{|c|c|c|c|c|c|c|}
\hline \multicolumn{2}{|c|}{ Mole Fraction } & \multirow[b]{2}{*}{ 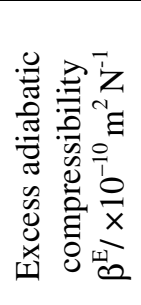 } & \multirow[b]{2}{*}{ 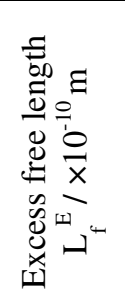 } & \multirow[b]{2}{*}{ 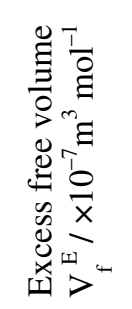 } & \multirow[b]{2}{*}{ 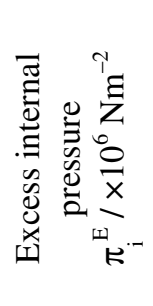 } & \multirow[b]{2}{*}{ 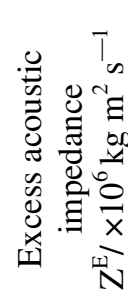 } \\
\hline $\mathrm{X}_{1}$ & $\mathrm{X}_{3}$ & & & & & \\
\hline \multicolumn{7}{|c|}{ System-I - 1-propanaol + toluene + dimethylacetamide (DMA) } \\
\hline 0.0000 & 0.6001 & 1.5410 & 0.0595 & -0.0396 & -274.22 & -0.1933 \\
\hline 0.0998 & 0.5002 & 1.2885 & 0.0509 & -0.0639 & -312.70 & -0.1658 \\
\hline 0.2000 & 0.4000 & 14 & 0.0408 & -0.1 & -356.56 & -0.1 \\
\hline 0.3 & 00 & 54 & 07 & -0. & -398.81 & -0 . \\
\hline 0.3999 & 0.2000 & 27 & 0.0205 & -0.1 & -441.87 & -0.0763 \\
\hline 0.5000 & 0.1000 & 0.2 & 0.0086 & -0.1720 & -450.14 & -0.0450 \\
\hline 0.6001 & 0.0000 & 0.1146 & 0.0048 & -0.1857 & -526.29 & -0.0293 \\
\hline \multicolumn{7}{|c|}{ System-II - 1- Butanol + toluene + dimethylacetamide (DMA) } \\
\hline 0.0000 & 0.5999 & 1.3485 & 0.0530 & -0.4929 & -264.06 & -0.1747 \\
\hline 0.0998 & 0.5000 & 05 & 0.0461 & -0. & -289.89 & -0.1511 \\
\hline 0.1998 & 0.4004 & 0.9378 & 0.0372 & -0.6740 & -319.68 & -0.1245 \\
\hline 0.3035 & 0.2918 & 0.7171 & 0.0287 & -0.6599 & -349.20 & -0.0955 \\
\hline 0.4000 & 0.2000 & & 0.0041 & -0.5604 & -380.48 & -0.1698 \\
\hline 0.5000 & 0.0998 & & 0.0129 & -0.5048 & -410.31 & -0.0473 \\
\hline 0.6001 & 0.0000 & 0.1706 & 0.0065 & -0.5314 & -435.98 & -0.0260 \\
\hline \multicolumn{7}{|c|}{ System-III - 1-propanaol + toluene + dimethylacetamide (DMA) } \\
\hline 0.0000 & 0.6099 & 1.1620 & 0.0543 & -0.6784 & -268.13 & -0.1860 \\
\hline 0.0999 & 0.5000 & 1.0924 & 0.6716 & -0.6716 & -286.38 & -0.1439 \\
\hline 0.2010 & 0.3971 & 0.9269 & 0.0031 & -0.5171 & -313.52 & -0.1218 \\
\hline 0.3000 & 0.3000 & 0.7569 & 0.0274 & -0.4182 & -450.63 & -0.0992 \\
\hline 0.4000 & 0.1997 & 0.5959 & 0.0233 & -0.3434 & -367.36 & -0.0755 \\
\hline 0.4900 & 0.0998 & 0.3879 & 0.0096 & -0.7698 & -389.21 & -0.1087 \\
\hline 0.6000 & 0.0000 & 0.2944 & 0.0107 & -0.5261 & -405.28 & -0.0306 \\
\hline
\end{tabular}

\section{Conclusion}

By analyzing exhaustively, the present investigation reveals that there exists a weak molecular association is identified. Mixing of DMA with 1-alkanols causes dissociation of 
hydrogen bonded structures of 1-alkanols. It is also observed that further addition of DMA with the mixture not only causes dissociation of hydrogen boded structures of 1alkanols but also a decrease in molecular association between toluene and 1-alkanols. The evaluated excess values too clearly predict weak molecular interactions existing between DMA-1-alkanols as well as toluene-1-alkanols.

\section{References}

1. Kincaid J F and Eyring H, J Chem Phys., 1937, 5, 587

2. Mehta S K and Chauhan R K, J Solution Chem., 1997, 26, 295

3. Dewan R K, Mehta S K, Parashar R and Bala K, J Chem Soc Faraday, Trans., 1991, 87, 1561.

4. Tabhane V A, Acoust Lett (G.B)., 1983, 6, 120.

5. Srinivasalu U and Ramachandra Naidu P, Indian J Pure Appl Phys., 1991, 29, 576.

6. Kannappan A N and Rajendran V, Acustica, 1991, 75, 192.

7. Isht Vibhu, Amit Misra, Manisha Guptha and Shukla J P, Pramana J Phys., 2004, 62(5), 1147.

8. Arul and Palaniappan L, J Acoust Soci India, 2000, 28, 393.

9. Pondey J D and Shukla A K, J Pure Appl Ultras., 1997, 15, 37.

10. Rowlison J S, Liquid and Liquid mixtures, $2^{\text {nd }}$ Edn., Butter Worths, London, 1969, 159.

11. Anwar Ali, Anil Kumar and Abida, J Chinese Chem Soc., 2004, 51, 477.

12. Pandey J D, Srivatsava V N P, Vyas V and Panta N, Indian J Pure Appl Phys., 1987, 25, 467.

13. Rai R D, Shukla R K, Shukla A K and Pandey J D, J Chem Thermodyn., 1989, 21, 125.

14. Kannappan A N and Rajendran V, Indian J Pure Appli Phys., 1991, 29, 465.

15. Tiwari K, Patra C and Chakravathy V, Acoust Lett., 1995, 19, 53.

16. Kannappan A N and Palanniappan L, Indian J Phys., 1999, 73B, 531.

17. Eyring H and Kincaid J F, J Chem Phys., 1938, 6, 620.

18. Nikam P S, Kapade V M and Mehdim Hasan, J Pure Appl Phys., 2001, 39, 433.

19. Rastogi M, Awasthi A, Guptha M and Shukla J P, Asian J Phys., 1998, 7, 739.

20. Ali A, Hydar S and Nain A K, Indian J Phys., 2000, 74B(1), 63.

21. Thirumaran S and Earnest, Jayakumar J, Indian J Pure Appl Phys., 2009, 47, 265.

22. Vaslman I I and Barkawitz M L, J Am Chem Soc., 1992, 114, 7889.

23. Anwar Ali, Anil Kumar Dinesh Chand and Bhajan Lal, Indian J Pure Appl Phys., 2003, 41, 928.

24. Palaniappan L, "Ultrasonic Investigation of Three Component Liquid Systems Rock Specimen and Diabetes Mellitus Incidence", Ph.D. Thesis, Annamalai University, 1998.

25. Rajendran V, Indian J Pure Appl Phys., 1994, 32, 523.

26. Rastogi M, Awasthi A, Guptha M and Shukla J P, Asian J Phys., 1998, 7, 739.

27 Sridevi U, Samatha K and Visvanantasarma A, J Pure Appl Ultras., 2004, 26, 1.

28. Rita Mehra and Meenakashi Pancholi, Indian J Pure App Phys., 2007, 45, 580. 


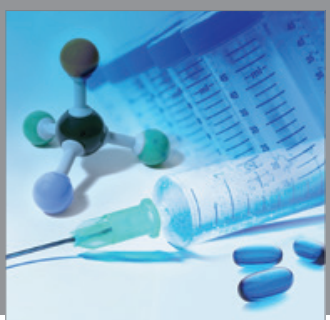

International Journal of

Medicinal Chemistry

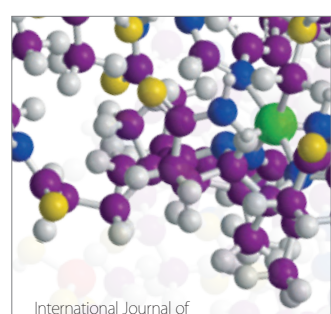

Carbohydrate Chemistry

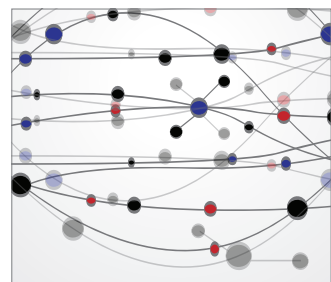

The Scientific World Journal
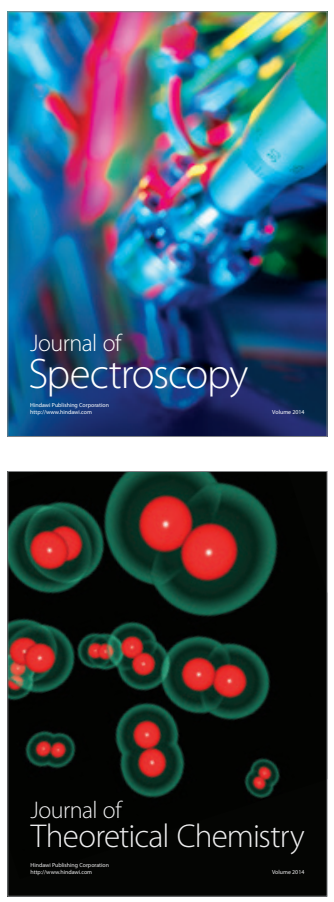
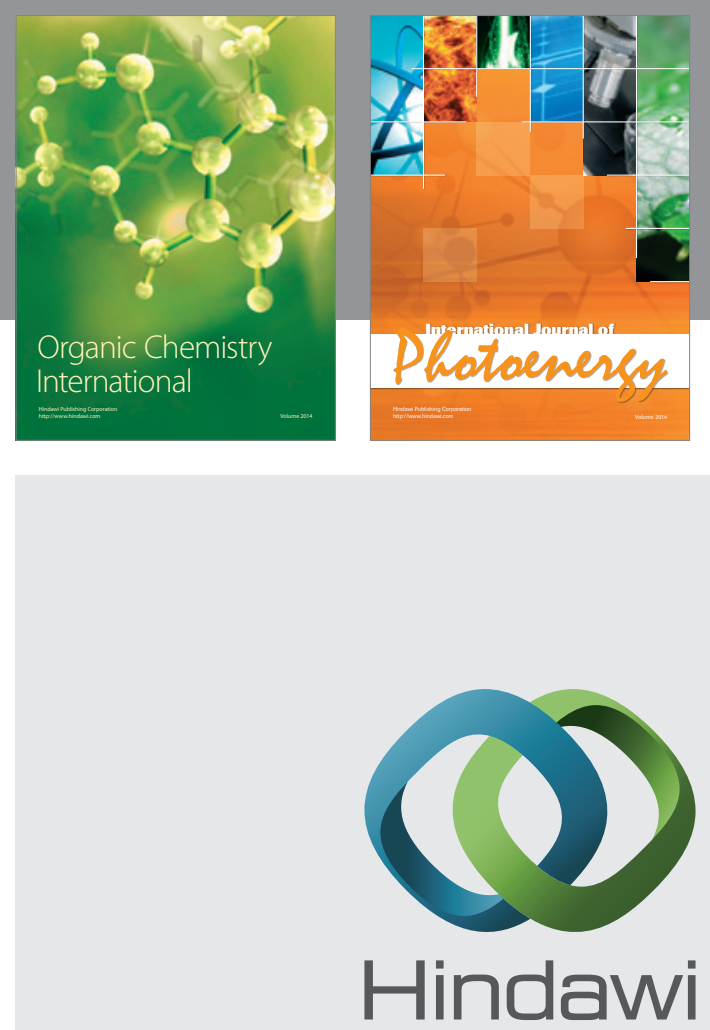

Submit your manuscripts at

http://www.hindawi.com
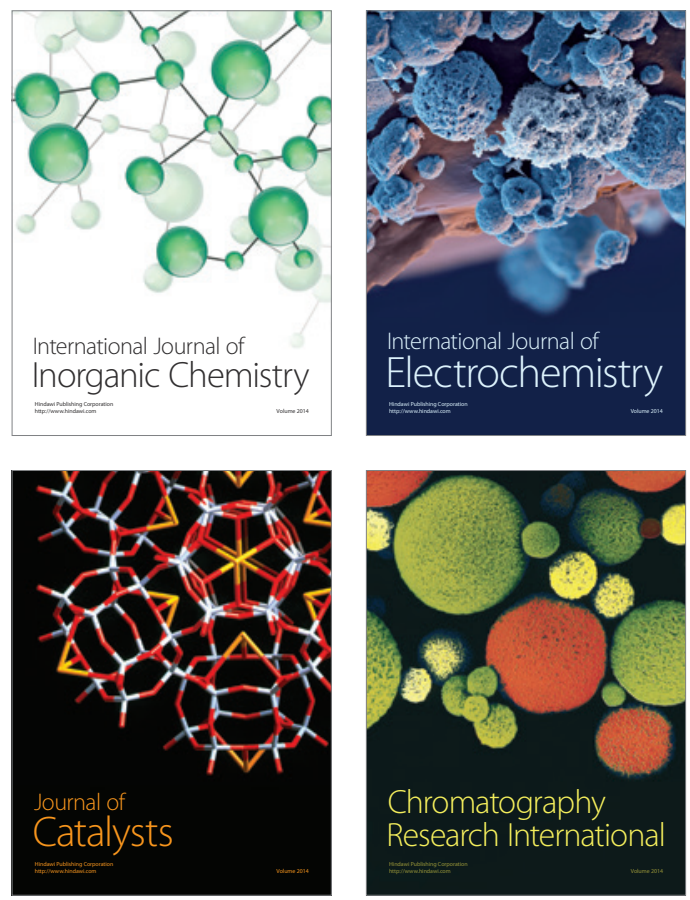
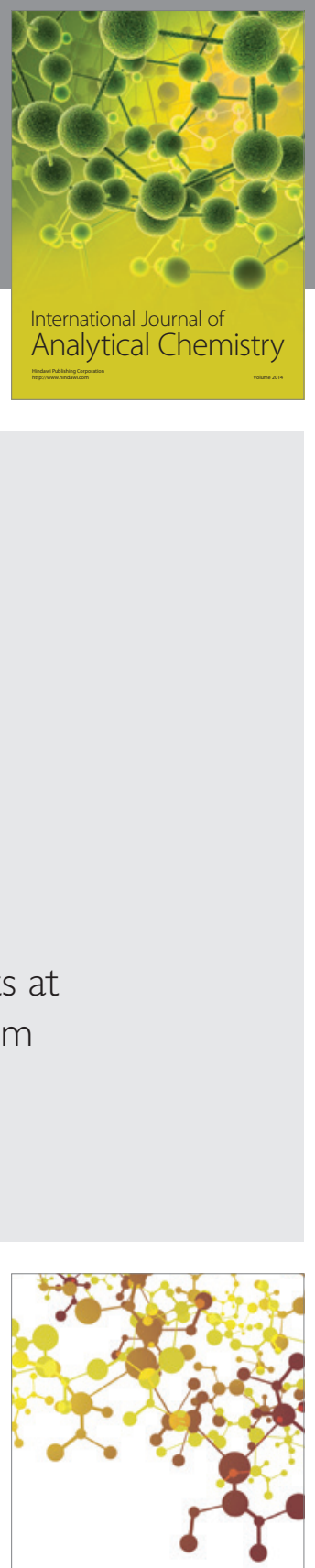

Journal of

Applied Chemistry
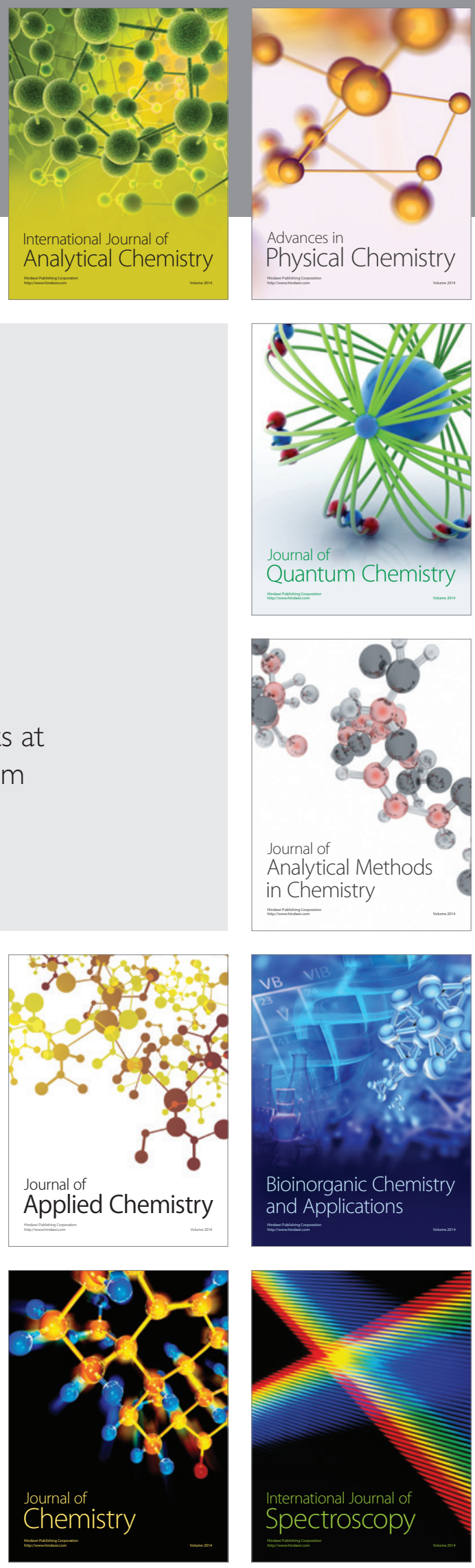